\title{
Visualising the number of people who cannot perform tasks related to product interactions
}

\author{
S. D. Waller, M.D. Bradley, P.M. Langdon, P.J. Clarkson \\ Engineering Design Centre, University of Cambridge, CB2 1PZ \\ $+44(0) 1223766961$ \\ sdw32@cam.ac.uk
}

Full Citation

Waller, S. D., Bradley, M. D., Langdon, P. M., \& Clarkson, P. J. (2013). Visualising the number of people who cannot perform tasks related to product interactions. Universal Access in the Information Society, 12 263-278.

\begin{abstract}
Understanding the number of people who cannot perform particular tasks helps to inform design decisions for mainstream products, such as the appropriate size and contrast of visual features. Making such informed decisions requires a dataset that is representative at the level of a national population, with sufficient scope and granularity to cover the types of actions associated with product use. Furthermore, visualisations are needed to bring the dataset to life, in order to better support comparing the number of people who cannot perform different tasks. The 1996/97 Disability Follow-up Survey remains the most recent Great British dataset to cover all types of ability losses that may be relevant to using everyday products. This paper presents new visualisations derived from this dataset, which are related to vision, hearing, cognition, mobility, dexterity and reach. Compared to previous publications on this dataset, the new visualisations contain task descriptions that have been simplified, described pictorially and separated out into different categories. Furthermore, two-dimensional visualisations are used to present exclusion results for products that require vision and / or hearing, and for tasks that require each hand to do different things. In order to produce these new visualisations, the publicly available version of this dataset had to be reanalysed and recoded, which is described here in detail.
\end{abstract}

Keywords Inclusive design, usability data, product assessment

\section{Introduction}

Everyone experiences some degree of ability loss, at various points in life and in various different situations. Minor difficulties might include understanding speech against background noise or picking up heavy objects. Major ability losses might include an inability to hear loud speech in a quiet room or lift a mug of coffee. Any kind of ability loss can impact product use, and the extent of this impact depends upon the type and severity of loss, the product and the real-world context. Well-designed products are easy to use by people with a wide range of abilities. Other products can unnecessarily cause frustration and difficulty even for those with minor ability losses.

Inclusive design promotes mainstream products that are usable by as wide a range of people as possible, without requiring specialised adaptations [5]. People with minor ability losses often continue to 
struggle on with mainstream products, perhaps due to the price and stigma associated with 'assistive' alternatives [8]. Improving the inclusivity of mainstream products therefore has great potential to improve quality of life across the population.

The wider remit of inclusive design includes issues of social exclusion, prior experience and culture, but here the focus is on capability loss and its consequence based on the percentage of British adults excluded from a range of tasks that are relevant to product use.

Mainstream products are readily available "off-the-shelf" in competitive markets and produced according to economies of scale. However, there is an inevitable limit to the level of ability loss that can be accommodated by mainstream designs, whilst keeping the production volume and styling suitable for mass-market sales at an appropriate price point. Therefore, it is often appropriate to set targets for the range of ability levels that an inclusive design will accommodate. This requires understanding the variation of ability in the population and how this affects interacting with products.

Users have differing characteristics to designers, and representing the diverse range of user characteristics has proved problematic in both research and practice. The complexity and mathematical nature of typical data sources limits their uptake amongst visually-oriented designers ([10], [17]). Engineering anthropometrics is one established approach that uses population data to inform design ([21], [22]). However, anthropometric and biomechanical data sources often draw together averages and percentiles from different data sources. This makes them unsuitable for predicting the percentage of adults excluded from a particular product, because several measurements or capabilities are often employed in combination when performing real-world tasks [15].

'HADRIAN' is a database of measurements for 100 real people which is integrated together with a holistic task assessment tool, and attempts to move anthropometrics beyond averages and percentiles. [15]. The computer aided design tool can predict how many of the people in its database would be unable to perform tasks that require the user to reach their hands to particular locations within an environment [20]. Although its modelling techniques and measurements are sophisticated, HADRIAN's sample was deliberately targeted to represent particular ability losses, so it cannot provide a population-based perspective.

This paper uses a nationally representative dataset to present a visual approach to understanding how many people are unable to perform particular actions. This is referred to here-in as the relationship between demand and exclusion, which is now described in more detail.

\subsection{Understanding demand and exclusion}

Using products typically requires several different capabilities. Considering a simple example, a mobile phone will typically require a certain level of vision ability to see the screen, and a certain level of 
dexterity ability to press the keys. Some people may be excluded because they cannot see the screen, others because they cannot press the keys, and some people will be unable to see the screen and also unable to press the keys.

People are excluded from using a product if any of their capabilities are less than those demanded by the product, given the real-world context [19]. Demand and exclusion is a way of presenting population data so that it directly reflects the number of people who would be excluded, based on the task that needs to be performed.

In combination with other methods, such as user involvement [3], and expert appraisal [18], visualisations of demand and exclusion can help to provide a population-based evaluation of a product. The visualisations of demand and exclusion presented here-in are intended to support:

- Communicating different types of ability loss and their prevalence;

- Making informed choices regarding the range of abilities that a new product design should attempt to accommodate;

- Evaluating the number of people who are excluded from particular aspects of product use;

- Prioritising design improvements that will make the most difference to the most people.

The target audience therefore includes industrial designers, engineers, project managers and design educators. The data presented here can be interpreted to help understand how many people would not be able to perform particular tasks of interest. For example, the data presented here regarding the number of people excluded from reading ordinary newsprint can help to understand how many people would be unable to distinguish a power button on a mobile phone. However, this interpretation process requires background knowledge regarding the type of ability variation being considered and the corresponding impact on real-world tasks, so is best supported by experts such as physiotherapists, ergonomists, and accessibility consultants.

A population-based understanding of demand and exclusion requires a dataset that is representative at a national level, which is best collected by sampling households at random. Performing real-world tasks with products often involves using vision, hearing, cognition, mobility, dexterity and reach. A single dataset is needed that covers all of these categories, in order to:

- Understand the relative prevalence of difficulties across the different categories;

- Understand the prevalence of co-occurring ability losses;

- Evaluate tasks that require multiple capabilities;

- Determine how many people could be included by different design changes.

Furthermore, the dataset needs to be in a generic format that enables comparative evaluation of different products. However, Johnson et al. [14] found that no currently available Great British dataset 
meets these criteria, and the best available dataset is the Office of National Statistics 1996/97 Disability Follow-up Survey (DFS) ([9], [12]).

Previous publications by the same authors have presented visualisations of demand and exclusion produced from derived variables that are within the original dataset ([6], [13], [24], [27]). However the visualisations presented here-in were produced following the identification of additional insights available from the original answers that participants gave in this survey, especially with respect to:

- Vision: separating out reading text and recognising a friend;

- Hearing: separating out understanding speech and hearing sounds;

- Cognition: separating out holding attention, concentrating, processing information, communicating and remembering;

- Mobility: separating out walking, steps and balancing;

- Dexterity: separating out the dominant and non-dominant hands, each with respect to grasping and fine-finger manipulation;

- Reach: separating out reaching above the head, out in front and down to the floor.

Separating out these different subcategories is particularly beneficial for product assessments, as it enables population data to be presented specific to the type of task being assessed [11]. Further details on this survey are now presented, followed by the procedure for reanalysis. Finally, visualisations of the reanalysed data are presented, and the process of producing these visualisations is discussed.

The visualisations of the reanalysed data presented here are further developed with respect to previously published versions ([25], [26]), specifically regarding the breakdown of dominant and nondominant hands in Fig. 8, and the bubble plots in Figs 10-12 that show exclusion arising from different demand combinations.

\section{Reanalysing participants' answers to the Disability Follow-up Survey}

\subsection{Further detail on the Disability Follow-up Survey}

The Disability Follow-up Survey (DFS) was commissioned to measure the prevalence of disability among adults in Great Britain, in order to plan welfare support. Disability was measured according to the severity of quality of life impairment. Approximately 7200 participants were asked up to 300 questions regarding their ability to perform different everyday tasks, such as: 
"Can you pick up a safety pin with your left hand?"

"Can you tie a bow in laces or string without difficulty?"

The questions were grouped together in 13 ability categories, 7 of which are most relevant for product interaction. The original survey authors named these categories: 'seeing', 'hearing', 'intellectual function', 'communication', 'locomotion', 'reach \& stretch', and 'dexterity'. Within each ability category, a panel of approximately 100 judges created a scale that related participants' survey question answers to their corresponding severity of quality of life impairment for that category [16].

Not all participants were asked all the questions. Instead the questions asked depended on each participant's previous answers, so that fewer questions were required to determine the severity of quality of life impairment. For example, if a participant indicated that they "cannot walk at all" then they were not asked "can you manage one step?". This example will be considered in further detail later.

The complete set of survey questions and the criteria that determined which questions were asked are publicly available [9]. Also available is a capability database that contains the 7200 participants' answers to each question and their corresponding severity of quality of life impairment.

\subsection{Reconstructing the answers to un-asked questions}

In order to use the DFS participants' original responses for predicting exclusion, it was first necessary to reconstruct the answers for all instances of missing data. In most cases, this missing data was caused by questions being skipped as the survey designers thought the answer was obvious from a previous question. In these cases, the answer to the previous question can provide an assumed answer for the skipped question. A detailed example of these assumed responses is provided in Table 1 for the question "can you manage one step on your own?".

Occasionally, there were still instances of missing data that were not caused by the control logic for the survey, in which case the answers were reconstructed by assuming the participant was "fully able", which is a conservative approach given the objective is to calculate exclusion.

Furthermore, participants' responses to the dexterity related questions were recoded into dominant or non-dominant hand, based on the specific question "are you left-handed, right-handed, or ambidextrous?". For this purpose, ambidextrous was assumed equivalent to right-handed, as this was the simplest assumption that allowed the data to be recoded.

\subsection{Creating new scales for demand}

The reconstruction process created approximately 100 new binary variables. Each binary variable indicates whether each survey participant was able, or unable to perform a particular task. 
The number of people who were unable to perform each of these 100 separate tasks could be presented directly. However, understanding the prevalence of different types of ability loss is much easier if these variables can be grouped together into a smaller number of ordinal demand scales. The process for testing variables to see if they can be grouped together is now explained in more detail.

\subsubsection{Defining a demand scale}

A demand scale is defined by a set of real-world tasks that demand increasing levels of user capability to perform. Tasks within a single scale must require the same type of user capability. In order for the real world tasks to successfully form a demand scale, everyone who cannot achieve a lower demand level task should also be unable to achieve each higher demand level task.

Interacting with products often requires the use of both hands, where each hand has to do different things. Users in the population may have different types of injury that independently affect the functional ability of each hand, so each hand was considered as a separate functional entity when creating the demand scales associated with dexterity and reach.

Some of the tasks within the DFS are obviously suitable for forming a demand scale, for example "read a newspaper headline", "read a large print book" and "read ordinary newsprint" clearly form a scale of increasing demand. Everyone who "cannot read a newspaper headline" will obviously also be "unable to read a large print book". However, it was often unclear whether particular dexterity related tasks might form a scale, so a procedure was constructed to verify the acceptability of a demand scale.

\subsubsection{Verifying the acceptability of a demand scale}

Demand scales were created from the 100 binary variables by first using expert judgement to identify sets of three tasks in the survey that were of a similar type and increasingly difficult to perform. The scale was then considered acceptable if over $80 \%$ of the participants who were unable to perform each task at lower demand levels were also unable to achieve each of the tasks at higher demand levels.

The acceptability threshold of $80 \%$ was judged to be an appropriate balance between ensuring the data is not unduly distorted by presenting it as an ordinal scale, and reducing the complexity of using the data for predicting exclusion. Similarly, choosing three tasks to define each scale was judged to be the best trade-off between the simplicity of the data presented and its granularity. Furthermore, when constructing the demand scales, some of the original DFS tasks had to be reworded to remove any mention of difficulty, as discussed further in Appendix A.

Considering an example within 'dexterity', the tasks involved in the DFS questions included picking up and carrying a mug, a pint of milk, a paperback book, a safety pin, a bag of potatoes, and a full kettle; 
turning a tap; turning the pages of a book; squeezing water from a sponge; unscrewing the lid of a coffee jar; using a pen or pencil; wringing out light washing; using scissors; and tying a bow in laces.

For this example, the phrase "with the dominant hand" will be shortened to DH. The tasks of "picking up a mug (DH)", "picking up a pint of milk $(D H)$ " and "picking up a bag of potatoes $(D H)$ " were judged as being likely to form a set of three tasks at increasing levels of demand. Figure 1 shows the crosstabulation results from these three binary variables, presented as a Venn diagram.

To check that these three variables formed an acceptable scale of increasing demand, the following percentages were checked:

- Of the 310 participants who reported that they “cannot pick up a mug (DH)”, what proportion of them also reported that they "cannot pick up a pint of milk $(D H)$ '? From Fig.1, (279+3) I $310=91 \%$.

- Of the 659 participants who reported that they "cannot pick up a pint of milk (DH)”, what proportion of them also reported that they "cannot pick up a bag of potatoes (DH)"? From Fig. $1,(279+373) / 659=98.9 \%$.

These tasks were therefore considered acceptable for creating a scale, because both these percentages were over $80 \%$. Following confirmation of the scale acceptability, all the participants who indicated that they "cannot pick up a mug $(\mathrm{DH})$ " were then recoded so that they also "cannot pick up a pint of milk $(D H)$ ", and all participants who indicated that they "cannot pick up a pint of milk $(D H)$ " were recoded so that they also "cannot pick up a bag of potatoes $(\mathrm{DH})$ ". Checking that the scale acceptability is over $80 \%$ ensures this recoding process does not unduly distort the original data.

\subsection{Calculating population exclusion}

The DFS dataset contains each participant's answer to each question, together with a weighting factor that depicts the number of GB adults that each participant represents. The number of GB adults who could not perform each of the tasks in the survey was therefore predicted by adding together the weighting factors for each of the survey participants who could not perform that task. The percentage of adults who could not perform each of the tasks in the survey was then calculated based on 43.3 million GB adults living in private households in 1997 [12].

The survey was conducted by random sampling of private households, obtained by filtering the Royal Mail Postcode Address File by mail volume. This sampling does not include people living in care homes, because the volume of mail received by care homes is not distinguishable from other business addresses. Note that the assumption of 43.3 million adults living in private households represents a refined value compared to previous publications by the same authors ([6], [13], [24], [25], [26], [27]), which calculated prevalence statistics based on 45.6 million adults living in the whole of Great Britain. Specifically, this 
means the percentages reported in Figures 2-10 are slightly higher than the equivalent percentages reported in [25] and [26]. For brevity, the phrase "percentage of adults" will be used throughout the rest of the paper to mean "percentage of the GB adult population living in private households in 1997".

Finally, the descriptions of the data points were rewritten to present demand and exclusion. Continuing the previous example, the percentage of adults who "cannot pick up a mug (DH)" is presented as being the percentage of adults excluded if they are "required to pick up a mug (DH)". Although the prevalence figures presented here-in could be adjusted based on the age and gender profile of Great Britain in 2010, this has not been performed in order to remain true to the original dataset. Comparing the results presented here against other countries or time periods requires making further assumptions regarding age and gender distributions, which is not recommended.

\subsection{Limitations of the analysis}

The demand scales in this paper are specifically defined as being ordinal, not interval. No judgement was made as to whether the tasks used to define the demand scales were in any way comparable across the different sets. Within each demand scale, no judgement was made as to whether the differences in ability required to perform the three tasks were in any way comparable. It is therefore not appropriate to label the points on the scales as low, medium or high demand.

The assumptions required to reconstruct the survey results were derived solely from the control logic that ran the original survey. The validity of these assumptions was not judged or tested in any way. Furthermore, some of the derived demand scales show an artificially high acceptability, because each participant was not necessarily asked all of the questions within the scale.

However, it is not considered prudent to further research the validity of the assumptions that were used to reconstruct the participants' responses, or further investigate the interaction between the acceptability of each scale and the control logic used to define the original survey. The DFS data is over 13 years old, and was collected for a different purpose.

The reanalysis presented here-in represents a transitional phase of research, as part of the wider $\mathrm{i} \sim$ design (2011) research programme ${ }^{1}$, whose primary objective is to enable better informed decisionmaking for inclusive product design. The research themes of this programme include:

- Designing a new survey specifically for the purpose of product evaluation and inclusive design;

- Developing and testing a method to assess products in order to estimate the number of people excluded;

- Producing software tools to support the use of survey data within an exclusion assessment.

\footnotetext{
${ }^{1}$ i design research programme website www-edc.eng.cam.ac.uk/idesign3/ Accessed August 2011
} 
The reanalysis of the Disability Follow-up Survey presented here-in was planned in order to support the research themes associated with developing the exclusion audit method and accompanying software [11], but also mindful of the need to design a new survey specifically for the purpose of estimating exclusion. Data from a 362 person pilot of the new survey has now been collected, and made publicly available [7]. IAlthough the authors are applying for additional funding to roll out their pilot survey on a nationally representative basis, in the meantime the Disability Follow-up Survey will remain the best nationally representative dataset to support exclusion calculations.

\section{Visualising exclusion for single demand scales}

The procedure outlined in Section 2 has enabled the demand scales in this paper to be categorised in the manner most suited for product evaluation, namely according to: vision, hearing, cognition, mobility, dexterity and reach. Note these categories are different from previous publications by the same authors, and the original survey. Appendix B explains these differences in further detail.

The reanalysis procedure calculates the proportion of GB adults excluded from a variety of tasks that were included within the 1996/97 Disability Follow-up Survey, and these results have been visualised in Figures 2 to 9. This data is intended to help understand whether one type of ability loss is particularly more prevalent than another, and identify instances where a slight change in the task that the user is required to perform will make a great difference to the number of people excluded. Although the absolute percentages have been included directly on the corresponding visualisations, relative comparisons between these results are expected to retain greater validity than the absolute numbers.

The insights gained from the demand and exclusion statistics are now discussed for each category in turn, followed by a discussion of the decisions made in constructing the visualisations, and prevalence comparisons with other sources. In all of the figures that present exclusion results, the calculated acceptability for each demand scale is printed next to it.

\subsection{Vision}

For vision, demand scales were created from tasks within the survey that referred to reading text and recognising shapes, as shown in Figure 2. The percentage of adults excluded from "reading ordinary newsprint" is approximately three times larger than the percentage excluded from "reading large print text", yet there is almost no difference in exclusion if the text size is increased from large print to a newspaper headline. This indicates considerable advantage for setting a minimum text size that is comparable to large print, yet diminishing returns for making it larger still. 


\subsection{Hearing}

For hearing, demand scales were created from tasks within the survey that referred to hearing speech and detecting sounds, as shown in Figure 3. The percentage of adults excluded from "following $a$ conversation against background noise" is much greater than the percentage excluded from any of the other tasks related to hearing, and also, much greater than the percentage excluded from any of the tasks related to vision.

\subsection{Cognition}

None of the DFS questions that related to cognition were suitable for creating ordinal demand scales, so the percentage of adults unable to perform these tasks is plotted on 13 separate axes, shown in Figures 4, 5 and 6. Figure 4 shows exclusion for tasks that require the user to hold attention, concentrate and process information, and indicates that a relatively small percentage of adults were excluded from “counting well enough to handle money", whereas a much greater percentage were excluded from "doing something without forgetting what the task was whilst in the middle of it”. Figure 5 shows exclusion for tasks that require the user to communicate with others, where the top four scales relate to separate aspects of communication, namely reading, writing, speaking and understanding others. The bottom three scales relate to higher-level tasks that also involve memory, which cause more people to be excluded. Figure 6 shows exclusion for tasks specifically relating to memory, one of which refers to remembering to do something in the future, while the other relates to the recall of existing knowledge. The exclusion is roughly comparable for both of these memory-related tasks.

\subsection{Mobility}

For mobility, demand scales were created from tasks within the survey that referred to walking, managing steps, and balancing, as shown in Figure 7. Interestingly, the percentage of adults excluded from the tasks associated with walking and steps is significantly greater than any of the other scales presented in this paper. Of particular interest, the percentage of adults excluded from "managing 12 steps" decreases dramatically when a handrail is available.

\subsection{Dexterity}

For dexterity, demand scales were created from tasks within the survey that related to single-handed actions of grasping, and fine-finger manipulation; these results are presented separately for the dominant, and non-dominant hand within Figure 8. The fine-finger scale for the non-dominant hand is the only one in this paper defined with two, rather than three tasks. This was unavoidable because the scale had to be 
directly compatible with the scale for the dominant hand, yet no data was available for using a pen with the non-dominant hand. Note that exclusion results for two-handed tasks are presented within Section 4.

One would expect a person's dominant hand to be stronger, and have better motor control, yet the increased frequency of use also increases the likelihood of an injury that reduces functional ability. Nevertheless, results from this analysis indicate that exclusion is consistently higher for tasks if they have to be performed by the non-dominant hand. Interestingly, the percentage of adults excluded from the picking-up related tasks was roughly equivalent to the percentage excluded from the fine-finger manipulation related tasks.

\subsection{Reach}

Demand scales were created for the dominant hand reaching up, both hands reaching up, and both hands reaching down, as shown in Figure 9. The percentage of adults excluded from tasks involving both arms was approximately twice the percentage excluded from tasks that only involved the dominant arm. Notably, the percentage of adults excluded from the easiest reaching-down related task of "bend down to touch the knees" is greater than the exclusion for the hardest tasks within vision, cognition, and reaching above the head.

\subsection{Constructing the visualisations}

Figures 2 to 9 were constructed in keeping with Tufte's [23] rules for visualising ratio-level data, which include:

- Numerical magnitude proportionately translated into visual length;

- Consistent Y axis scale across all visualisations, with zero point shown in all cases

- Datapoints labelled directly on the visualisation;

- Different areas distinguished by shading, rather than crosshatching.

The distance of each data point from the zero level provides an absolute magnitude of the exclusion, and further shading was introduced to help visually identify the amount of difference between each data point. Furthermore, the thermometers were drawn around the data to convey the message that all the datapoints provided exist somewhere along a continuous scale, much like temperature. The entire section of the thermometers below the zero level was shaded differently from the datapoints, so that the visual areas presented are directly proportional to the exclusion magnitudes.

\subsection{Prevalence comparisons from other sources}

As mentioned in Section 2, it is notoriously difficult to compare the prevalence estimates presented here with other surveys conducted at different times and/or in different countries. Nationally 
representative sampling techniques are rarely used, other surveys use different threshold criteria to define their measurements, and the age/gender distribution of countries change over time.

Furthermore, the DFS set out to measure the number of people who self reported that they could not achieve particular real-world tasks. This is not directly compatible with other functional measures of performance, such as distance visual acuity or hearing threshold levels. Neither is it directly compatible with prevalence measures of conditions such as arthritis, because one such condition can cause many functional effects, and a person may have difficulty performing real-world tasks because of the compounding effect of several of their conditions. The survey presented here was conducted across Great Britain, whereas most comparable statistics are typically reported for the UK, which additionally includes Northern Ireland. Nevertheless, some UK prevalence statistics are now presented for the sake of completeness, and to illustrate the scope of different ways in which prevalence statistics are often reported.

Access economics [1] report cites $2.9 \%$ of the UK population with partial sight and blindness, defined as distance visual acuity worse than 6/12. Action on hearing loss [2] fact sheet presents $16.7 \%$ of UK population have some degree of hearing loss, defined as quietest detectable sound between 25 and $39 \mathrm{~dB}$. Arthritis Research Campaign [4] fact sheet indicates $29 \%$ of UK adults say they are currently affected by arthritis or joint pain, although the majority of these relate to self-reported joint and back pain. Also for comparison, Table 2 further presents some preliminary self-report results from the 362 person pilot survey [7] conducted as part of the i design (2011) research programme ${ }^{1}$; recall this pilot survey was introduced in Section 2.

\section{Visualising exclusion across two demand scales}

Performing real-world tasks with products typically involves a cycle where the user perceives, thinks and acts. This commonly requires vision, hearing, cognitive and dexterity ability. Although issues associated with cognition are a particularly significant cause of exclusion for product interactions, the treatment of cognition within the Disability Follow-up Survey is less applicable to product interaction than the other ability types, so will not be considered further.

In order to calculate exclusion for tasks that require combinations of abilities, each survey participant in the capability database is flagged if any of the demands exceed any of their abilities, and the weighting factors are added together for each of these flagged participants. This procedure is described in further detail within Waller et al. [27], and implemented within the freely available exclusion calculator [6]. Building on this approach, a software platform has been developed as part of the i design (2011) research programme ${ }^{1}$. This software platform can use the reanalysed dataset to calculate the exclusion associated with any network of tasks linked in any combination of 'AND ALSO' / 'OR'. This software platform was 
used to produce Figures 10 to 12, which show exclusion results for some particularly interesting simple examples, where the user is required to:

- Perceive text AND ALSO hear speech;

- Perceive text OR hear speech;

- Perform fine-finger manipulation with the dominant hand, AND ALSO perform grasping with the non-dominant hand.

Throughout Figures 10 to 12, the area of each circle is linearly proportional to the number of adults excluded. This conversion factor is identical across all the plots, enabling a visual comparison of the variation in exclusion across the different bubble plots [23]. The actual percentage of adults excluded is also printed beneath each circle. Insights from these visualisations are now presented in further detail, followed by discussion of the decisions made constructing the visualisations.

\subsection{Exclusion for perceiving text AND ALSO hearing speech}

Figure 10 shows the percentage of adults excluded from a task that requires the user to read text AND ALSO hear speech. Firstly, to provide a baseline for comparison, the column of bubbles on the far left hand side of Figure 10 give exclusion values for varying text sizes, when the task does not require the user to hear speech. These values are identical to the exclusion values for the scale on the left-hand side of Figure 2. Similarly, the bubbles in the bottom row of Figure 10 give exclusion values for varying speech sounds, when the task does not require the user to read text. These are identical to the exclusion values on the left-hand side of Figure 3.

The meaning of the exclusion values for tasks that require both these abilities is now presented by considering the example of using a mobile phone indoors, without background noise. This task typically requires the user to be able to read the numbers on the buttons, AND ALSO understand the speech from the earpiece. Assuming the numbers on the buttons are roughly equivalent to 'read ordinary newsprint', the speech is equivalent to 'use an ordinary telephone', and only considering exclusion due to vision and hearing in the first instance, Figure 10 predicts that the $4.92 \%$ of adults will be excluded from this task.

Furthermore, note from Figure 2 that $3.52 \%$ of adults would be excluded considering only the text, and from Figure 3 that $1.96 \%$ of adults would be excluded considering only the speech. The combined exclusion value of $4.92 \%$ correctly takes care of double counting for survey participants who have cooccurring vision and hearing losses, whereas a straight summation of the individual components would give an incorrect value of $5.48 \%(3.52+1.96)$.

Figure 10 can also be used to examine the potential benefit of a particular design change, in the context of co-occurring ability losses. For example, if the text on the buttons was made clearer, say equivalent to reading a large print book, Figure 10 predicts the exclusion would reduce $2.28 \%$ (down to $2.64 \%$ ). 
Alternatively, if the text on the buttons remained unchanged, but the earpiece was changed to a loudspeaker that was of equivalent clarity to someone talking loudly, exclusion would reduce $0.75 \%$ (down to 4.17\%). Again, note that these reductions in exclusion are slightly smaller than the reductions that would be predicted using the individual component scales, because some of the people who were excluded because of the vision demand, were also excluded because of the hearing demand.

\subsection{Exclusion for perceiving text OR hearing speech}

Figure 11 shows the percentage of adults who would be excluded from a task that can be completed either by reading text OR hearing speech. Considering another practical example, but only taking into account exclusion associated with vision and hearing in the first instance, a screen reader accessible website can be interpreted either by reading the text, OR by listening to the information through a screen reader. Assuming the text size can be adjusted so that it is equivalent to 'read a newspaper headline', and assuming the volume of the computer can be adjusted to be equivalent to 'hear loud speech in a quiet room' loud speech, Figure 11 predicts that only $0.1 \%$ of adults would be excluded from perceiving this information.

Providing information in alternative forms enables a dramatic reduction in exclusion. To consider some comparisons, if the 'headline size' website text was not screen reader accessible, Figure 2 predicts that approximately $0.74 \%$ of adults would be excluded (over seven times as many). Considering another example, if a website contains video content that is not described by a textual alternative, then assuming the visual demand is roughly comparable to 'read ordinary newsprint', and the hearing demand is roughly comparable to, 'use an ordinary telephone', Figure 10 predicts that approximately $4.92 \%$ of adults would be excluded. The video that requires 'Vision AND Hearing' therefore excludes 49 times more adults than the screen reader accessible website that requires 'Vision OR Hearing'.

\subsection{Exclusion for two-handed tasks}

Reanalysing the data and producing separate demand scales for the dominant and non-dominant hand has enabled significant advancement in the prediction of exclusion for two-handed tasks. It is now possible to predict exclusion when each hand is required to do different things, while retaining the integrity of the dataset that also contains data regarding vision, hearing, cognition, reach and mobility. Figure 12 shows one example plot that is possible from the reanalysis, where the dominant hand performs fine-finger manipulation, while the non-dominant hand performs grasping. These types of actions are particularly common when interacting with hand-held products, and opening packaging.

Similar to Figure 10, the column of bubbles on the far left of Figure 12 show exclusion values when the non-dominant hand has to perform grasping and the dominant hand does not have to do anything; 
these values match the bottom left scale in Figure 8. Furthermore, the bottom row of bubbles in Figure 12 show exclusion values when the dominant hand has to perform fine-finger manipulation and the nondominant hand does not have to do anything; these values match the top right scale in Figure 8.

To illustrate the use of Figure 12 to predict exclusion for tasks that require both hands, consider the example of opening the zip of a bag. Opening such a zip typically involves grasping the bag with the nondominant hand, approximately equivalent to 'pick up a pint of milk', and fine-finger manipulation from the dominant hand, approximately equivalent to 'pick up a safety pin'. Figure 12 therefore predicts that $4.58 \%$ of adults would be excluded from opening this zip.

Note that Figure 12 predicts exclusion for instances where the user is forced to use both hands to perform the task. It does not predict exclusion for instances when the user can choose a one-handed or a two-handed usage mode. This kind of calculation requires the software platform developed as part of the $\mathrm{i} \sim$ design (2011) research programme ${ }^{1}$. Nevertheless, ensuring that a design gives the user the choice of one-handed or two-handed usage is recommended as best practice.

\subsection{Constructing the visualisations}

Figures 10 to 12 shows the variation of exclusion with two dependent variables. This could be plotted as a three-dimensional surface or bar chart, a shaded contour plot or a bubble chart. The visualisations are intended to help the reader understand which design changes could make the most difference that the most people. For this purpose, the reader needs to identify ratio level changes in exclusion between the different data points (twice as much, three times as much etc.)

A three-dimensional surface or bar chart does not have a zero level baseline, which would significantly impede the reader in determining the amount that exclusion changes between the data points. Considering a shaded contour plot, the eye is very good at determining whether something is darker than something else, but not very good at determining how much darker it is [23].

Tufte [23] further notes that although the eye is not perfectly accurate at spotting proportional changes in the area of the circle, it is pretty good, and making the area linearly proportional to the exclusion is considered a better choice than making the radius linearly proportional. Furthermore, the actual exclusion values are printed underneath every circle, in order to help compensate for the inaccuracy of the eye when perceiving changes in area.

\section{Conclusions}

Despite being over 13 years old and collected for a difference purpose, the 1996/97 Disability Followup Survey remains the best currently available data source for predicting design exclusion in Great 
Britain. The reanalysis and visualisations presented within this paper have enabled specific improvements in the use of this dataset for understanding the relationship between demand and exclusion, especially through separating out:

- Cognitive related tasks associated with holding attention, concentration, processing information, communication and memory;

- Dexterity related tasks for the dominant hand and non-dominant hand;

- Mobility related tasks associated with walking, steps, and balancing.

Together with the software platform developed as part of the i design (2011) research programme ${ }^{1}$, the reanalysed dataset can be used to predict the exclusion for any combination of demands across the scales presented in this paper, linked together with any possible combination of the logical operators 'AND ALSO' / 'OR'. (The lead author can be contacted to perform these calculations on a consultancy basis). This paper has presented exclusion visualisations for some particularly interesting demand combinations, especially:

- Perceiving information using vision or hearing;

- Performing fine-finger manipulation with the dominant hand while grasping with the nondominant hand.

However, the i design (2011) research programme ${ }^{1}$ recognised that a new data source was needed specifically for the purpose of predicting exclusion, and therefore designed such a survey and conducted a 362 person pilot. The documentation and results for this survey are now publicly available [7]. Further research is now required to roll out the pilot on a national basis, and further develop and test the exclusion audit procedure and corresponding software to make the new data useful in practice.

\section{References}

[1] Access Economics (2009). Future Sight Loss UK 1: Economic Impact of Partial Sight and Blindness in the UK adult population. RNIB. PDF available online www.rnib.org.uk (accessed August 2011).

[2] Action on hearing loss (2011). Facts and figures on deafness and tinnitus. PDF available online www.actionhearingloss.org.uk (accessed August 2011).

[3] Aldersey-Williams, H., Bound, J., Coleman, R. (1999). The methods lab: User research for design. Design for Ageing Network. Available at www.education.edean.org (accessed August 2011).

[4] Arthritis Research Campaign (2007). Arthritis: the big picture. PDF available online www.ipsos-mori.com (accessed August 2011). 
[5] British Standards Institute (2005). British Standard 7000-6: 2005. Design management systems - Managing inclusive design - Guide.

[6] Clarkson, P.J., Coleman, R., Hosking, I., Waller, S.D. (2007). Inclusive design toolkit, Engineering Design Centre, University of Cambridge, UK. ISBN 9780954524326 Available at www.inclusivedesigntoolkit.com (accessed August 2011).

[7] Clarkson, P.J. et al. (2010), Towards Better Design, [computer file]. Colchester, Essex: UK Data Archive [distributor], May 2012. SN: 6997 , http://dx.doi.org/10.5255/UKDA-SN-69971. Survey data and documentation available from www.esds.ac.uk (accessed August 2012).

[8] Coleman, R. (2002). Living Longer: the new context for design, Design Council Policy Papers series, Design Council, London.

[9] Department of Social Security Social Research Branch (2000). Disability Follow-up to the 1996/97 Family Resources Survey [computer file]. Colchester, Essex: UK Data Archive [distributor], 3 March 2000. SN: 4090.

[10] Elton, E.M. and Nicolle, C.A. (2011). Translating inclusive capability for designers, INCLUDE 2011, Royal College of Art, 6th International Conference on Inclusive Design, 1820 April 2011, Helen Hamlyn Centre, RCA, London, UK, 2011, p1-10.

[11] Goodman-Deane J., Waller S.D., Williams E.Y., Langdon P.M., Clarkson P.J. (2011). Estimating Exclusion: A Tool to Help Designers. INCLUDE 2011, Royal College of Art, 6th International Conference on Inclusive Design, 18-20 April 2011, Helen Hamlyn Centre, RCA, London, UK.

[12] Grundy E., Ahlburg D., Ali M., Breeze E., Sloggett A. (1999). Research report 94: Disability in Great Britain, 1999 (Corporate Document Services, London).

[13] Keates and Clarkson (2004). Countering design exclusion. Springer, London UK ISBN 1852337699

[14] Johnson D., Clarkson P.J., Huppert F. (2009). Capability Measurement for Inclusive Design. Journal of Engineering Design 21(2/3), 275-288.

[15] Marshall, R., Case, K., Porter, J.M., Sims, R., Gyi, D.E. (2004). Using Hadrian for eliciting virtual user feedback in 'Design For All'. IMECHE Proceedings Part B: Journal of Engineering Manufacture, 218(B9), pp. 1203-1210.

[16] Martin, J. and Elliot, D. (1992). Creating an overall measure of severity of disability for the office of population census and surveys disability survey. J. Royal Statistical Society Series A, 155(1): 121-140. 
[17] Nickpour, F. and Dong, H. (2010). Developing user data tools: Challenges and opportunities. in Langdon P.M., Clarkson P.J., and Robinson P. (eds) Designing inclusive interactions. Springer, London UK.

[18] Nielsen, J. (1993). Usability Engineering, Morgan Kaufmann, San Francisco.

[19] Persad, U., Langdon, P., Clarkson P.J. (2007). Characterising user capabilities to support inclusive design evaluation. Universal Access in the Information Society, vol 6, no 2, pp 119135 .

[20] Porter, J.M., Marshall, R., Sims, R., Gyi, D.E., Case, K. (2006). Hadrian gets streetwise. In: Pikaar, RN, Koningsveld, EAP, Settels, PJM, (eds) Meeting Diversity in Ergonomics. Proceedings IEA 2006 Congress, Elsevier.

[21] Smith S., Norris B. Peebles L. (2000). Older Adultdata: The Handbook of Measurements and Capabilities of the Older Adult, (Department of Trade and Industry, London).

[22] Stanton N., Hedge A., Brookhuis K., Salas E. and Hendrick, H. (2004). Handbook of Human Factors and Ergonomics Methods (CRC Press).

[23] Tufte, E.R. (2001). The Visual Display of Quantitative. Information, 2nd edn, Graphics Press, ISBN 0-9613921-4-2.

[24] Waller, S.D. and Clarkson, P.J. (2009). Tools for inclusive design. In Stephanidis C. (ed.) The Universal Access Handbook. Taylor and Francis, London, UK. ISBN 9780805862805.

[25] Waller, S.D., Williams, E.Y., Langdon, P.M., Clarkson P.J. (2010a). Understanding the cooccurrence of ability loss. In Langdon P.M., Clarkson P.J., and Robinson P. (eds) Designing inclusive interactions. Springer, London UK.

[26] Waller, S.D., Williams, E.Y., Langdon, P.M., Clarkson P.J. (2010b). Quantifying exclusion for tasks related to product interaction. In Langdon P.M., Clarkson P.J., and Robinson P. (eds) Designing inclusive interactions. Springer, London UK.

[27] Waller, S.D., Langdon, P.M., Clarkson P.J. (2010c). Using disability data to estimate design exclusion. Int. J. Universal Access Information Society 9(3):195-207. Available online at www.springerlink.com. DOI 10.1007/s10209-009-0168-x. 


\section{Appendix A}

An ideal data source for supporting design decisions would be able to predict and compare the percentage of the population who are excluded from a particular task, and the percentage who would have difficulty. Unfortunately, apart from the questions that asked about reaching out in front and above the head, the DFS did not take a consistent approach to difficulty when phrasing the questions asked. Some of the questions asked "can you...", while others asked "do you have difficulty doing...".

The authors experience teaching people to use demand scales for task assessments found that inconsistent mentions of difficulty within some, but not all of the statements describing a demand scale can cause people to completely misinterpret the scale. The authors therefore had no choice but to remove any mention of difficulty from statements describing the demand scales. Specifically, this affects the following statements

- Figure 2: 'Read ordinary newsprint', 'recognise a friend across the road ';

- Figure 3: 'Follow a conversation against background noise', 'hear loud speech in a quiet room';

- Figure 8: 'Use a pen'.

The equivalent data points in Figs 10-12 are similarly affected. When participants answered the questions regarding reaching out in front and above the head, they could report that the task was not difficult, difficult or impossible. In this paper, exclusion results are presented only using the participants who indicated that the task was impossible, in order to provide a consistent approach throughout all the statistics presented here-in.

\section{Appendix B}

The process of reanalysing the original DFS dataset has enabled the data presented in this paper to be clustered in a manner that is more suitable for product assessments, but note the clustering and naming used here is different from previous publications by the same authors, and the originally published survey results. Specific differences are now discussed in turn:

Cognition: The 13 tasks presented in Figures 4, 5 and 6 were originally contained within categories named as "thinking" and "communication" in previous publications by the same authors. This distinction between thinking and communication was based on the categorisation from Grundy et al. (1996), yet the distinction was not considered beneficial for product assessments, and the reconstruction process enabled all 13 questions to be combined into one category. 
Dexterity: The tasks presented in Figure 8 show exclusion results for tasks involving the dominant hand, and separately involving the non-dominant hand. Figure 12 shows exclusion for tasks involving both hands, where each hand is doing something different. The dexterity results presented here-in are completely different from any previous publications by the same authors, which used different tasks from the survey to define the scales, and predicted exclusion for tasks that could be performed with either hand, and tasks where both hands had to do the same thing. The format of presentation within this paper is considered more useful for predicting exclusion for the kind actions that have to be performed when using typical products.

Mobility and Reach: The tasks presented in Figure 7 associated with walking, steps and balancing have previously been presented in a category called "locomotion" in previous publications by the same authors, and within the originally published survey results.

In this paper, bending tasks are presented within "reach" (in Figure 9), whereas bending tasks have previously been presented within "locomotion" in previous publications by the same authors, and within the originally published survey results. In the context of a product assessment it makes more sense to present reaching down in the same category as reaching up.

Also within "reach", previous publications have presented the number of adults unable to reach one arm above their head, whereas the results here specifically consider the dominant arm, for better compatibility with the corresponding results for dexterity tasks. 


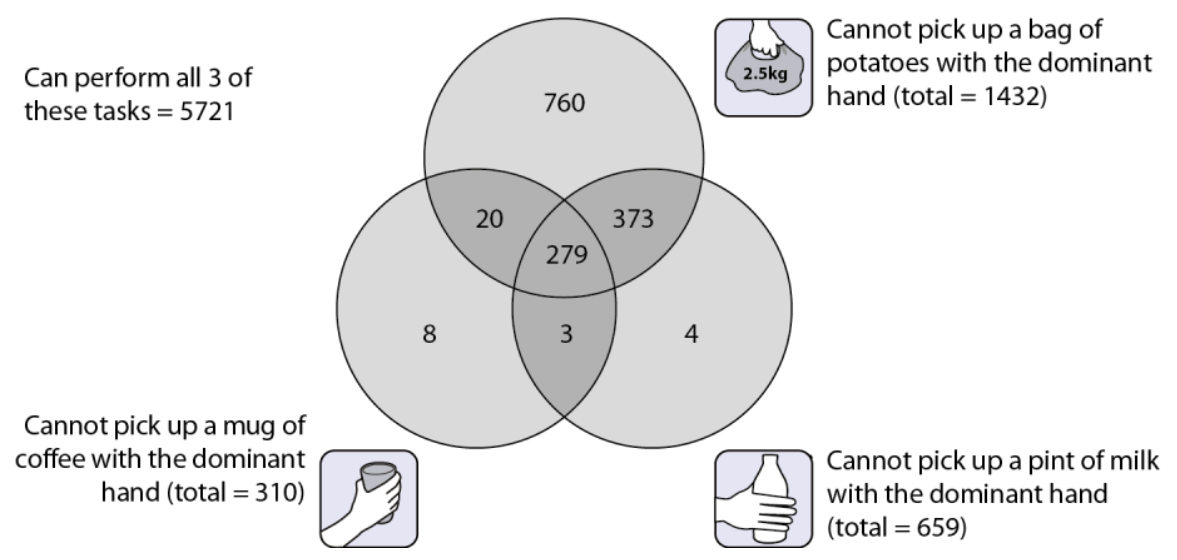

Fig. 1: Cross-tabulation results from the three binary variables associated with picking up a mug of coffee, a pint of milk and a bag of potatoes.
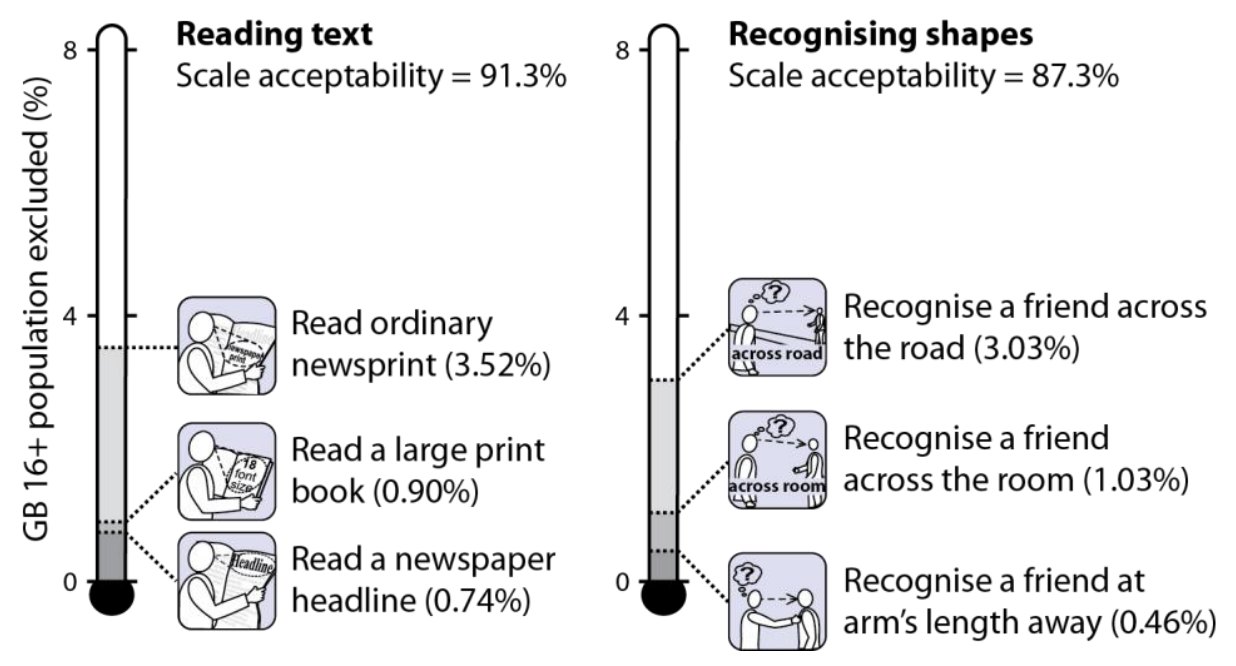

Fig. 2: The percentage of adults excluded for tasks that require the user to see. Exclusion statistics assume that vision aids are used. 

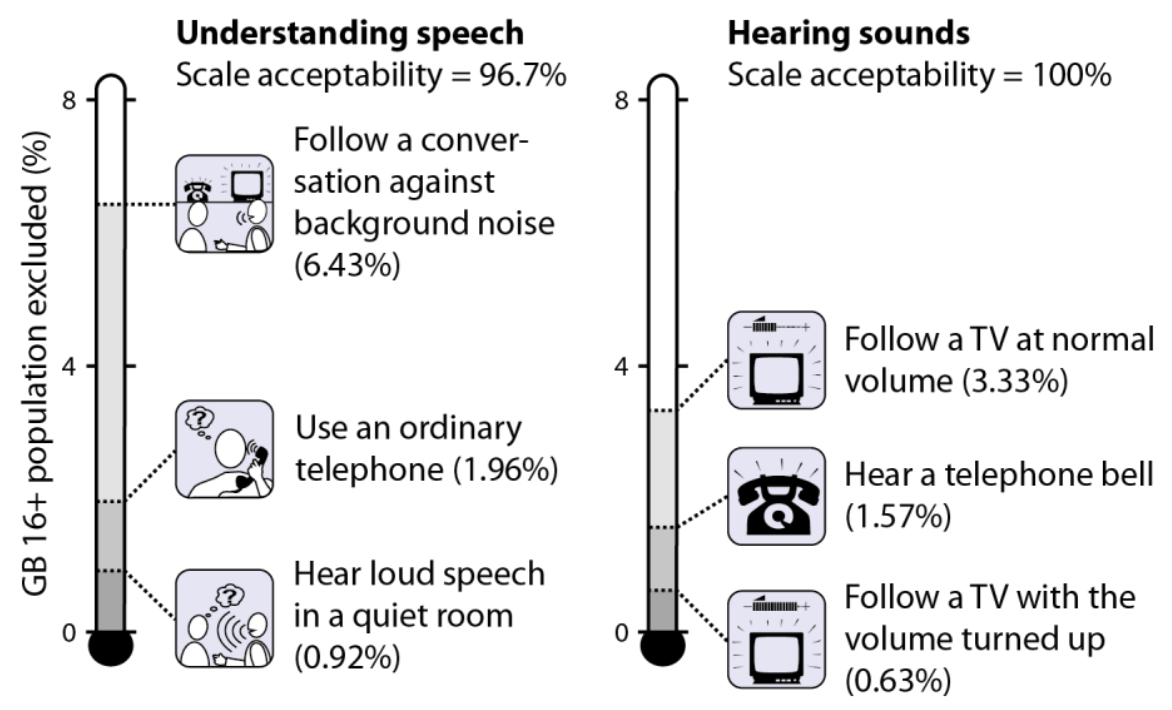

Fig. 3: The percentage of adults excluded for tasks that require the user to hear. Exclusion statistics assume that hearing aids are used.

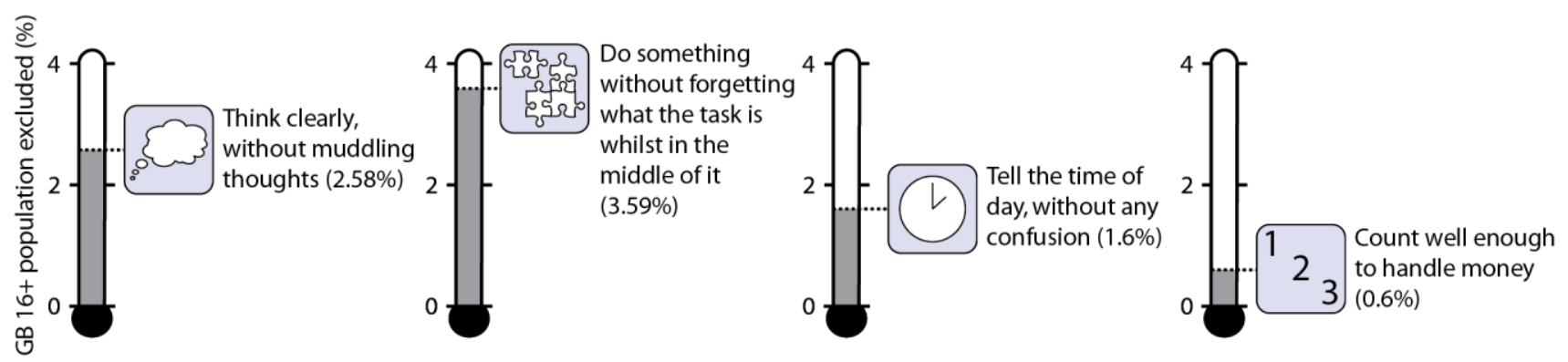

Fig. 4: The percentage of adults excluded for tasks that require the user to hold attention, concentrate and process information. 

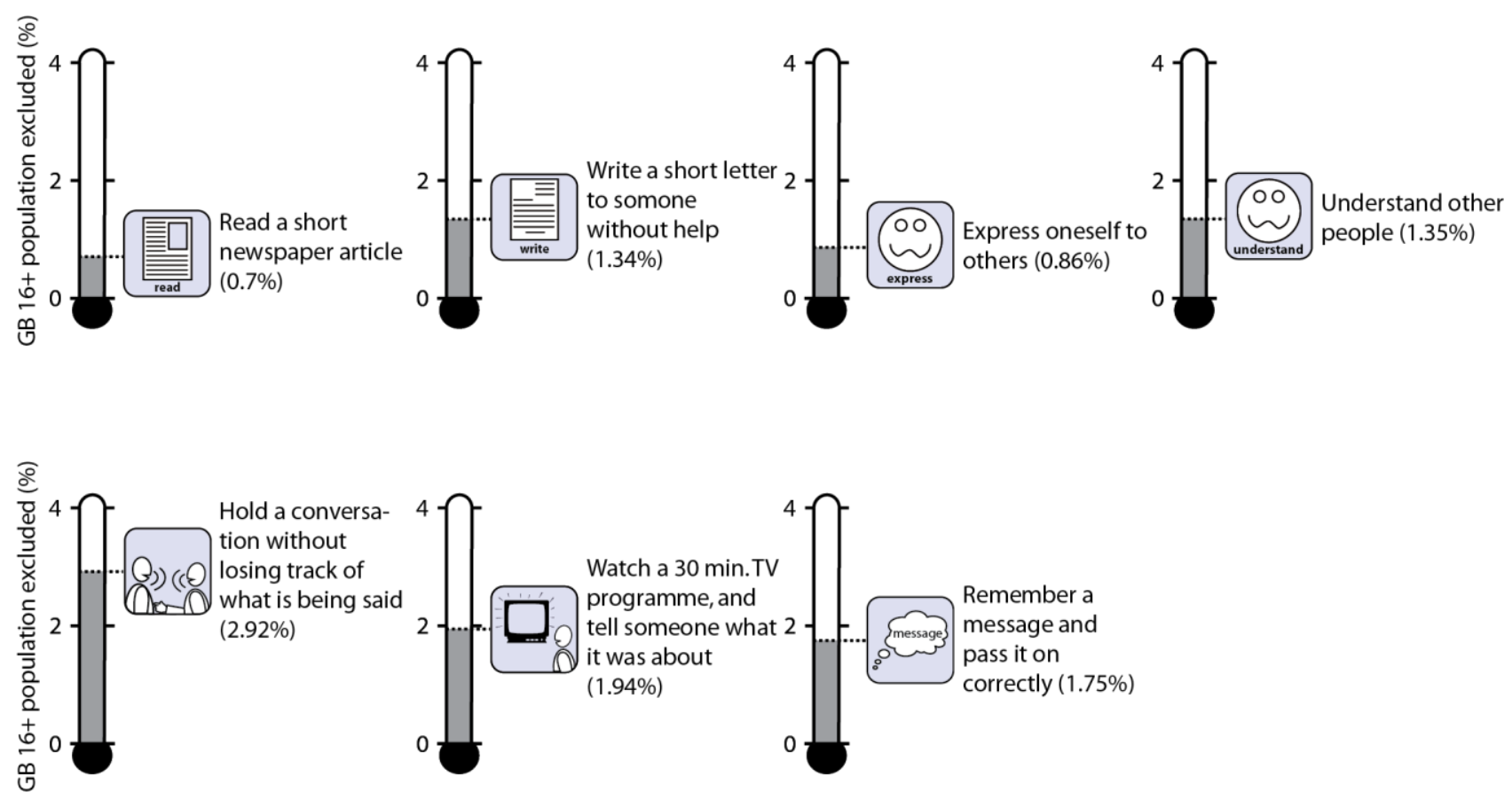

Fig. 5: The percentage of adults excluded for tasks that require the user to communicate with others.

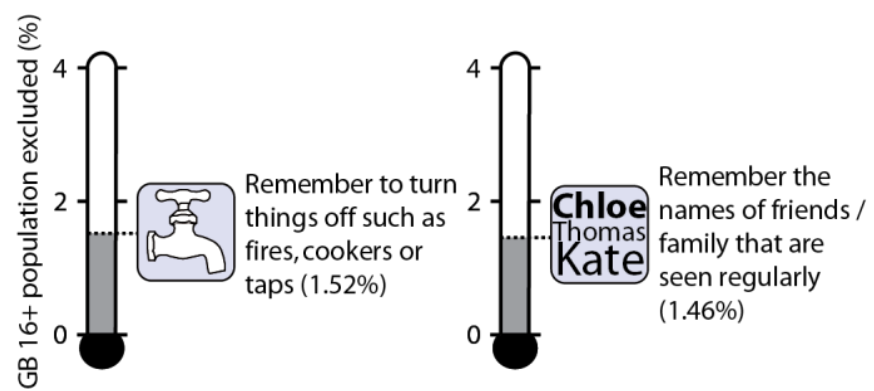

Fig. 6: The percentage of adults excluded for tasks that require the user to remember to do things in the future, or recall existing knowledge. 

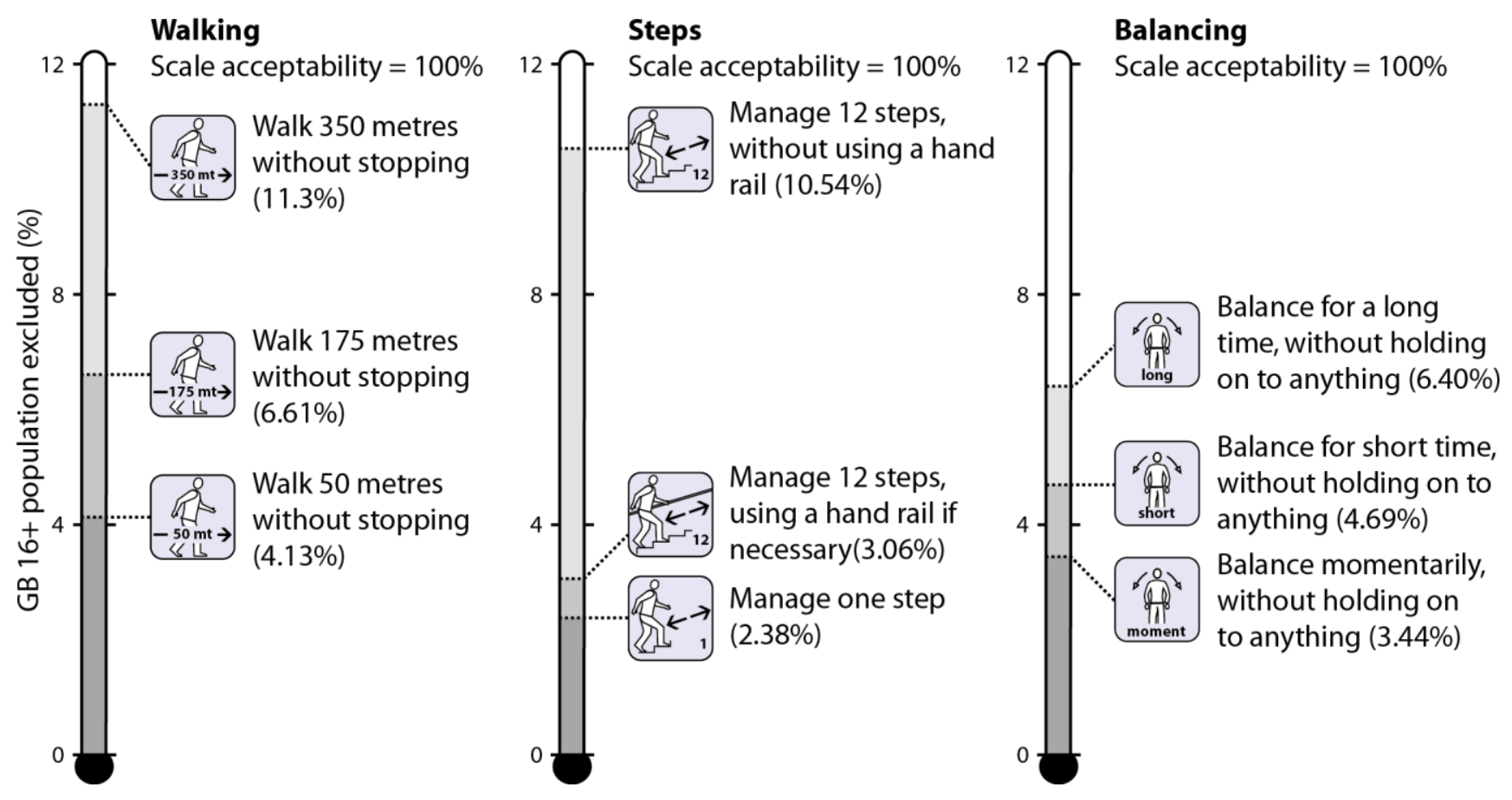

Fig. 7: The percentage of adults excluded for tasks that require the user to move around and balance. Exclusion statistics assume that mobility aids are used, but no one else is assisting. 

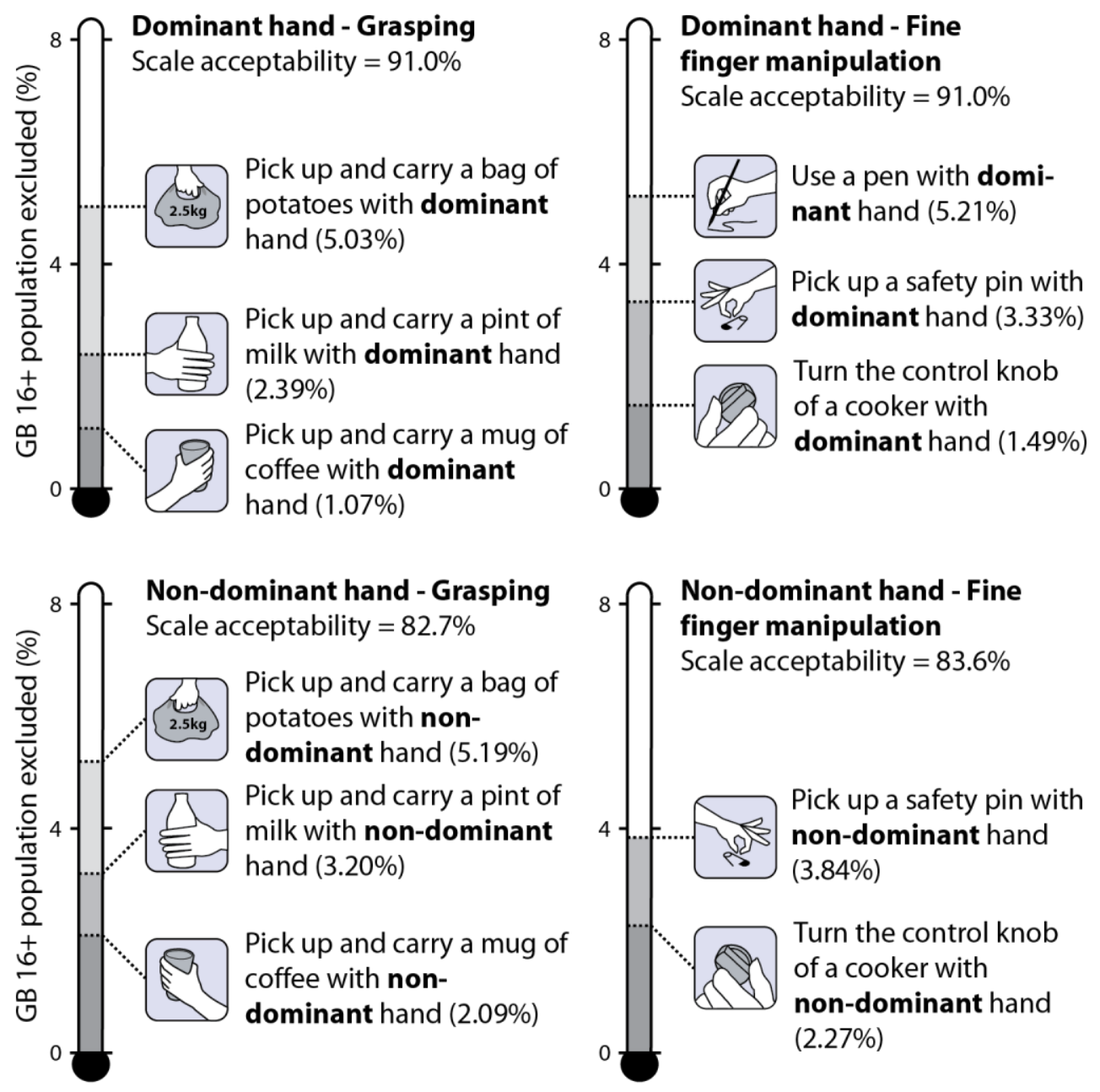

Fig. 8: The percentage of adults excluded for tasks that require the use of one hand. 

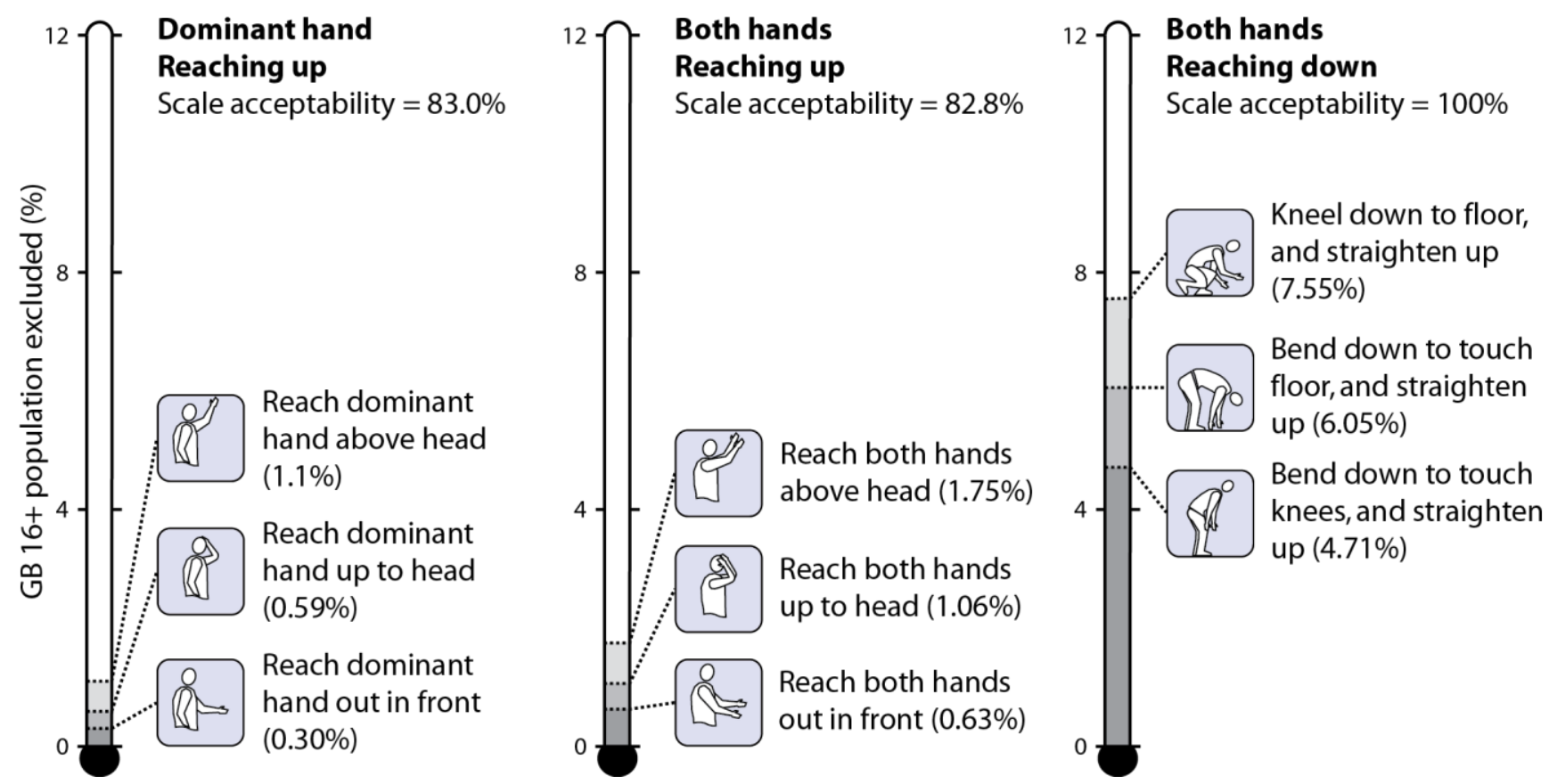

Fig. 9: The percentage of adults excluded for tasks that require the user to reach one or both hands to different positions. Exclusion statistics for bending assume that something is available to hold on to, but no one else is assisting. 


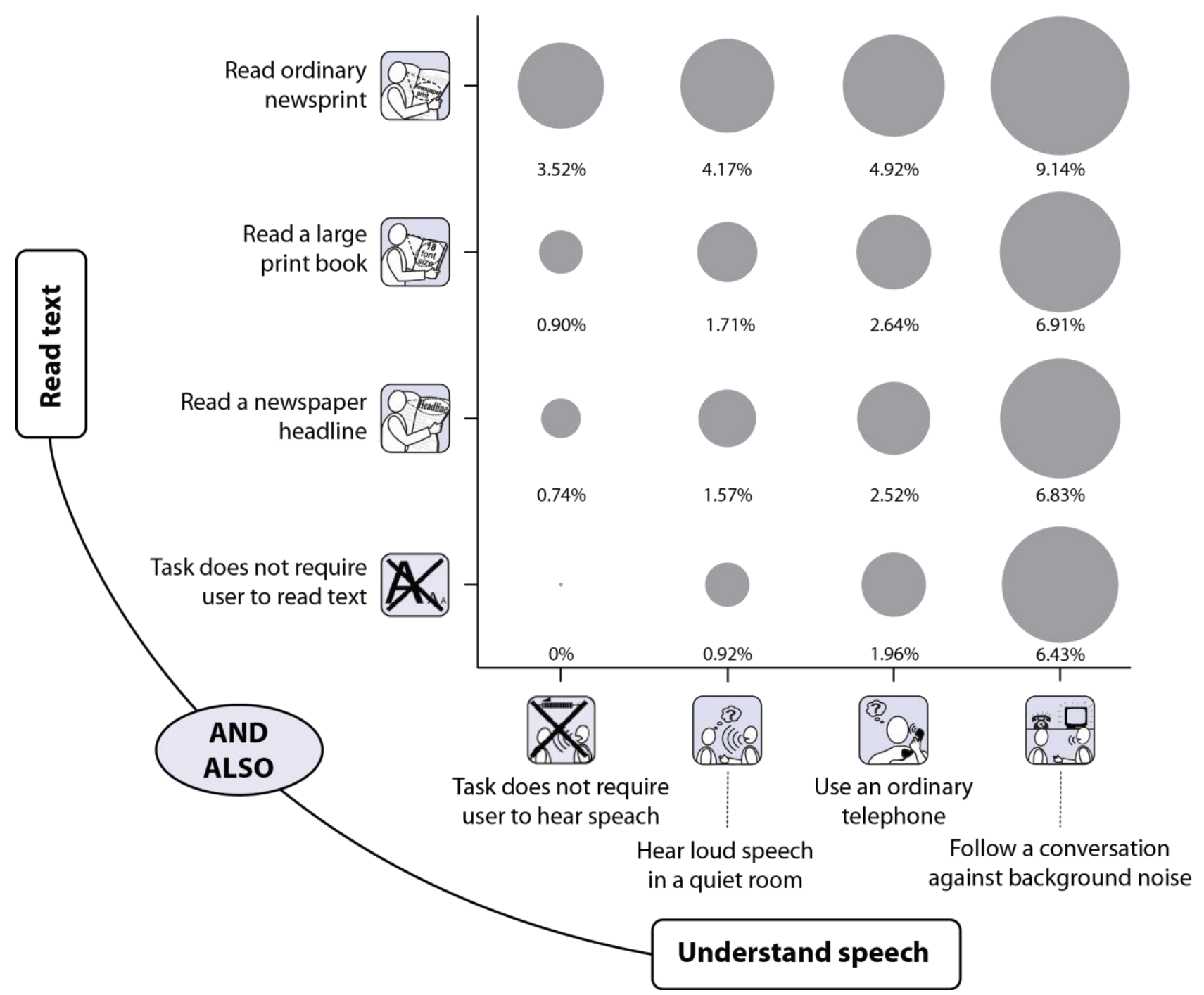

Fig. 10: Percentage of adults excluded for tasks that require the user to be able to read text, AND ALSO be able to understand speech. 


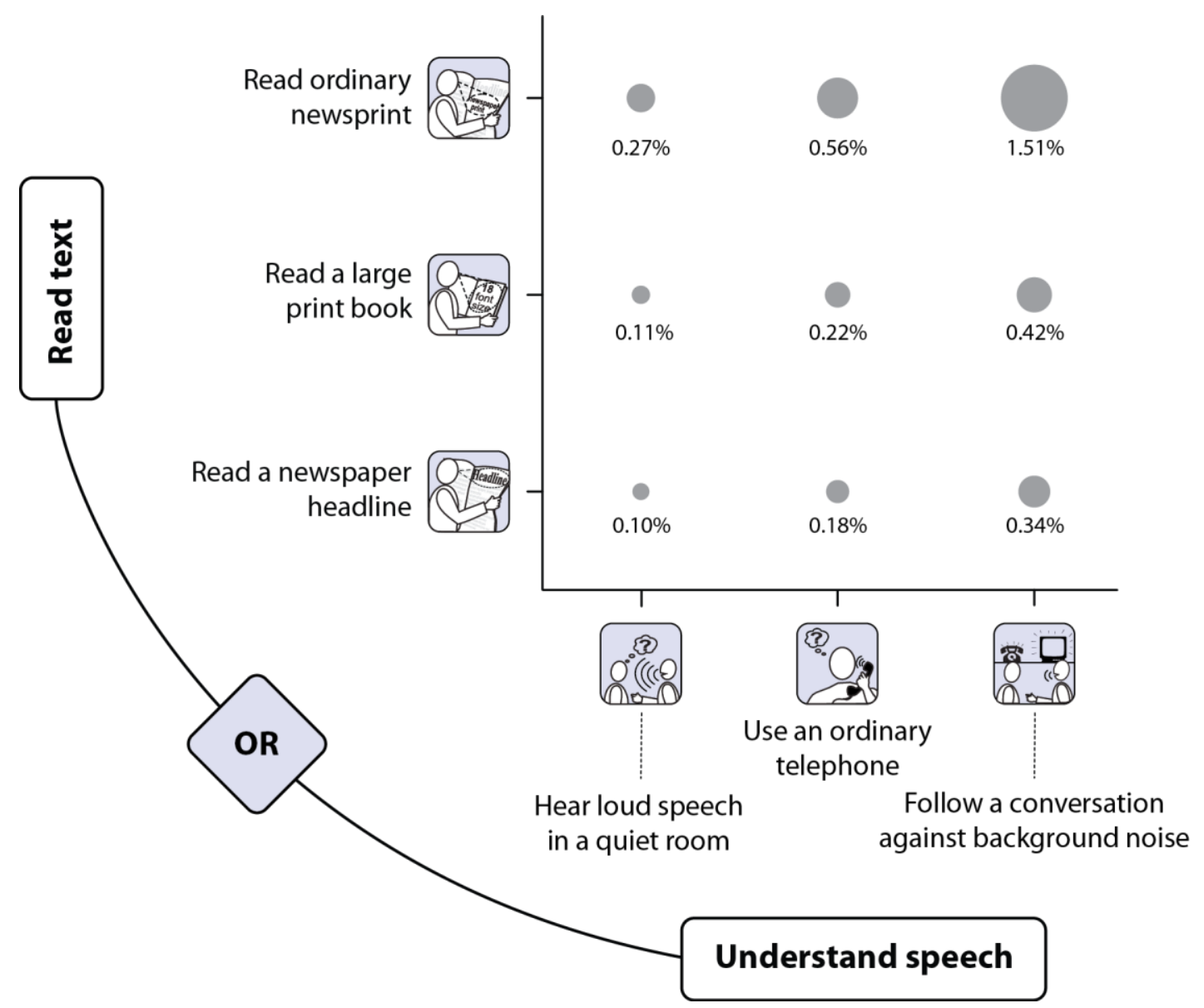

Fig. 11: Percentage of adults excluded for tasks that require the user either to be able to read text, OR be able to understand speech. 


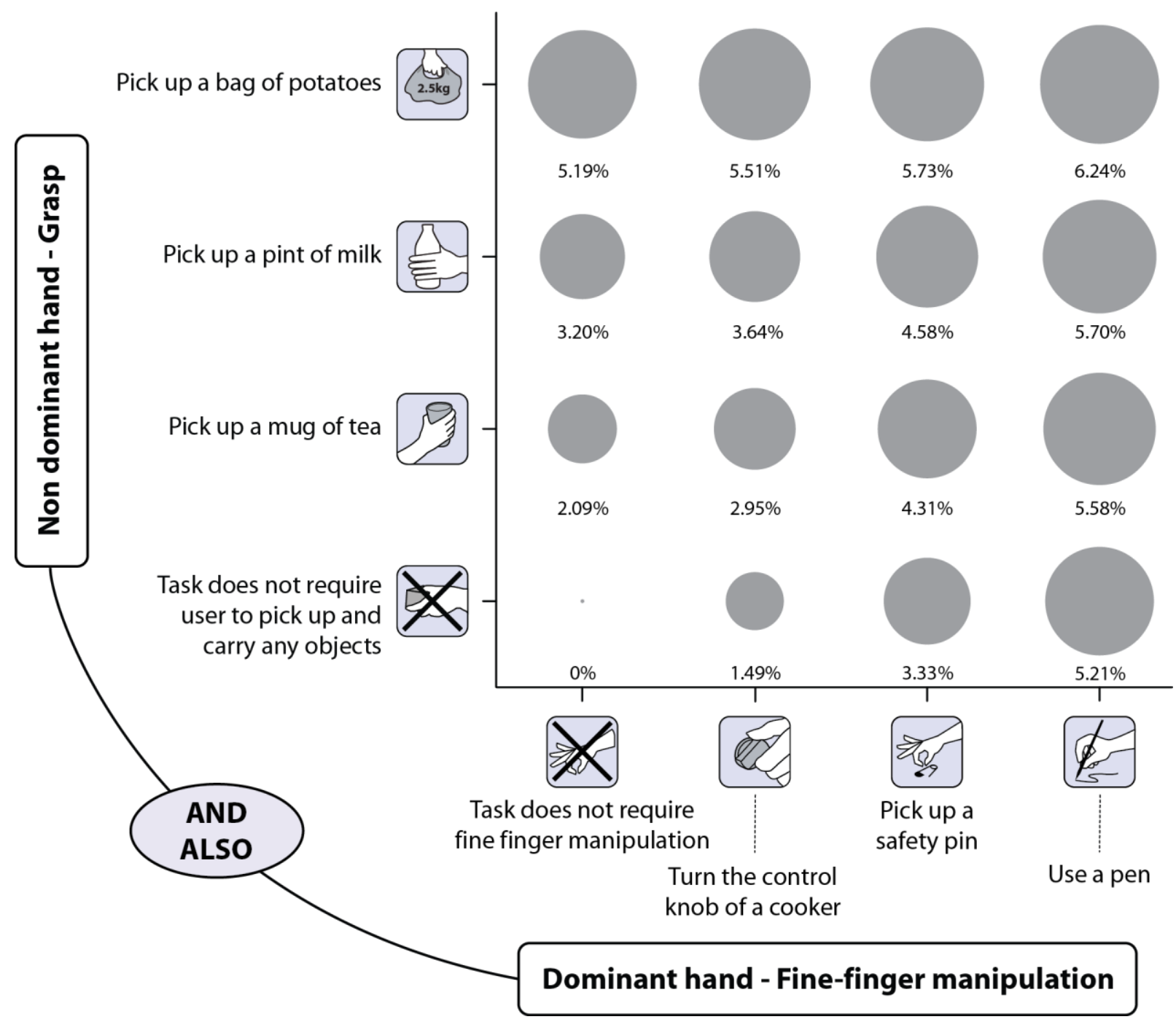

Fig. 12: Percentage of adults excluded for tasks that require the user to perform fine-finger manipulation with the dominant hand AND ALSO grasp an object with the non-dominant hand. 


\begin{tabular}{|l|l|l|c|}
\hline \multicolumn{1}{|c|}{ YES } & $\begin{array}{l}\text { No. of } \\
\text { people }\end{array}$ & \multicolumn{1}{|c|}{ NO } & $\begin{array}{c}\text { No. of } \\
\text { people }\end{array}$ \\
\hline $\begin{array}{l}\text { Directly asked this question, and } \\
\text { answered "yes" }\end{array}$ & 2661 & $\begin{array}{l}\text { Directly asked this question, and } \\
\text { answered "no" }\end{array}$ & 138 \\
\hline $\begin{array}{l}\text { Not asked because they "do not } \\
\text { have great difficulty walking up or } \\
\text { down steps or stairs" }\end{array}$ & 3809 & $\begin{array}{l}\text { Not asked because they "cannot } \\
\text { walk at all" }\end{array}$ & 131 \\
\hline Missing data recoded to fully able & 9 & $\begin{array}{l}\text { Not asked because they "can } \\
\text { only walk a few steps" }\end{array}$ & 420 \\
\hline Total & 6479 & Total & 689 \\
\hline
\end{tabular}

Table 1: Reconstructed results for "can you manage one step on your own?". 


\begin{tabular}{|l|c|c|}
\cline { 2 - 3 } \multicolumn{1}{l|}{} & $\begin{array}{l}\text { Somewhat or } \\
\text { very limited }\end{array}$ & $\begin{array}{l}\text { Not at all } \\
\text { limited }\end{array}$ \\
\hline $\begin{array}{l}\text { Because of your eyesight, how limited are you in doing your daily } \\
\text { activities? }\end{array}$ & $9.9 \%$ & $90.1 \%$ \\
\hline $\begin{array}{l}\text { Because of your hearing, how limited are you in doing your daily } \\
\text { activities? }\end{array}$ & $4.5 \%$ & $95.5 \%$ \\
\hline $\begin{array}{l}\text { Because of any problems with your mobility, how limited are you in } \\
\text { doing your daily activities? }\end{array}$ & $12.2 \%$ & $87.8 \%$ \\
\hline $\begin{array}{l}\text { Because of any problems with your hands, how limited are you in } \\
\text { doing your daily activities? }\end{array}$ & $11.1 \%$ & $88.9 \%$ \\
\hline $\begin{array}{l}\text { Because of any problems with reaching your arms above your head } \\
\text { or out to the sides, how limited are you in doing your daily activities? }\end{array}$ & $6.8 \%$ & $93.2 \%$ \\
\hline $\begin{array}{l}\text { Because of any difficulties with memory or concentration, how } \\
\text { limited are you in doing your daily activities? }\end{array}$ & $13.5 \%$ & $86.5 \%$ \\
\hline
\end{tabular}

Table 2: Self-report results from the 362 productive responses (out of 990 issued) from [7]. This survey postcode sampled adults living in private households within England and Wales. 